\title{
New pathological findings in emphysema of childhood: 2. Overinflation of a normal lobe
}

\author{
ALISON HISLOP and LYNNE REID \\ Department of Experimental Pathology, Institute of Diseases of the Chest, \\ Brompton Hospital, London S.W.3
}

\begin{abstract}
A quantitative study of the structure of the affected lobe from a patient with childhood lobar emphysema is here reported. The left upper lobe was removed from a child aged $2 \frac{1}{2}$ months who was suffering from acute respiratory distress and showing on his radiograph hypertransradiancy of the left lung.

At operation the lobe was found to be increased in volume and rotated through $180^{\circ}$ on its pedicle. After laboratory injection of some of the pulmonary artery branches and distension of the lobe, the stage of development of the various lung structures was studied quantitatively. The arteries and bronchi were normal in number and structure: the alveolar number was normal for the age of the child but the volume of an alveolus was increased four to five times, as was total lung volume.

The antenatal development of this lobe was normal and it seems that the emphysema could have followed the rotation of the lobar hilum, although no cause for this rotation was found.
\end{abstract}

In a recent article (Hislop and Reid, 1970) it was emphasized that emphysema in the newborn lung was of several different pathological types. A new type characterized by too many alveoli was reported-a polyalveolar emphysema-and methods were described of quantitating the growth of airways, alveoli, and arteries so that the pathogenesis of the disease could be more precisely judged. The same methods have been applied to a case of childhood lobar emphysema and the results are described below. Regarded clinically as similar to the case reported earlier, quantification of the resected lobe suggests that antenatal development must have been virtually normal.

\section{CASE HISTORY}

The patient was a male child born, after a normal pregnancy, at term and weighing $9 \mathrm{lb}$ (20 October, 1969). Apart from slight dyspnoea and feeding difficulties for the first five days, he showed no abnormality for two months, after which he caught a cold that developed into acute bronchiolitis which caused his admission to hospital. At that time his general condition was good and his respiratory rate normal; he was not cyanosed or distressed. The breath sounds were markedly diminished over the right lung: no other sounds were heard. There was slight subcostal recession. 'The electrocardiogram was normal.
$X$-ray examination (Fig. 1) showed that the dia $\stackrel{\AA}{\perp}$ phragm was in the normal position but that the hear $\vec{b}$ and trachea were displaced to the right. The left lung? was transradiant and the left hilum depressed with the vessels just visible although too small and reduced in number. The lower lobe was too far medial. further radiograph five weeks later was unchanged. $\bar{\partial}$

A diagnosis of left upper lobar emphysema waș made and left upper lobectomy was planned. Af thoracotomy this lobe was found to be three to four times the normal size, occupying the whole of the left hemithorax and encroaching on the right. The upper lobe appeared to have been rotated forwards and upwards $180^{\circ}$ around its pedicle so that the lingula lay at the apex of the chest. The lingula wass less distended than the superior segments of the upper lobe. When the surgeon replaced the lobe to its correct position its volume did not change ands the lobe was therefore removed. The left lower lobe was smaller than normal and though it inflated welR it only half-filled the left hemithorax. At the end of the operation there was still mediastinal displace ment to the right.

The postoperative course was uneventful and the baby remains well. A film taken eight weeks post operatively showed an apical pleural space and media- 0 stinal shift now to the left: the left lower lobe was aerated but smaller than would be expected. Fiv 8 months later the apical space has reduced in size so that aerated lung is visible between the diaphragm? at its normal level, and the second interspace an teriorly: the lung vessels appear normal. 


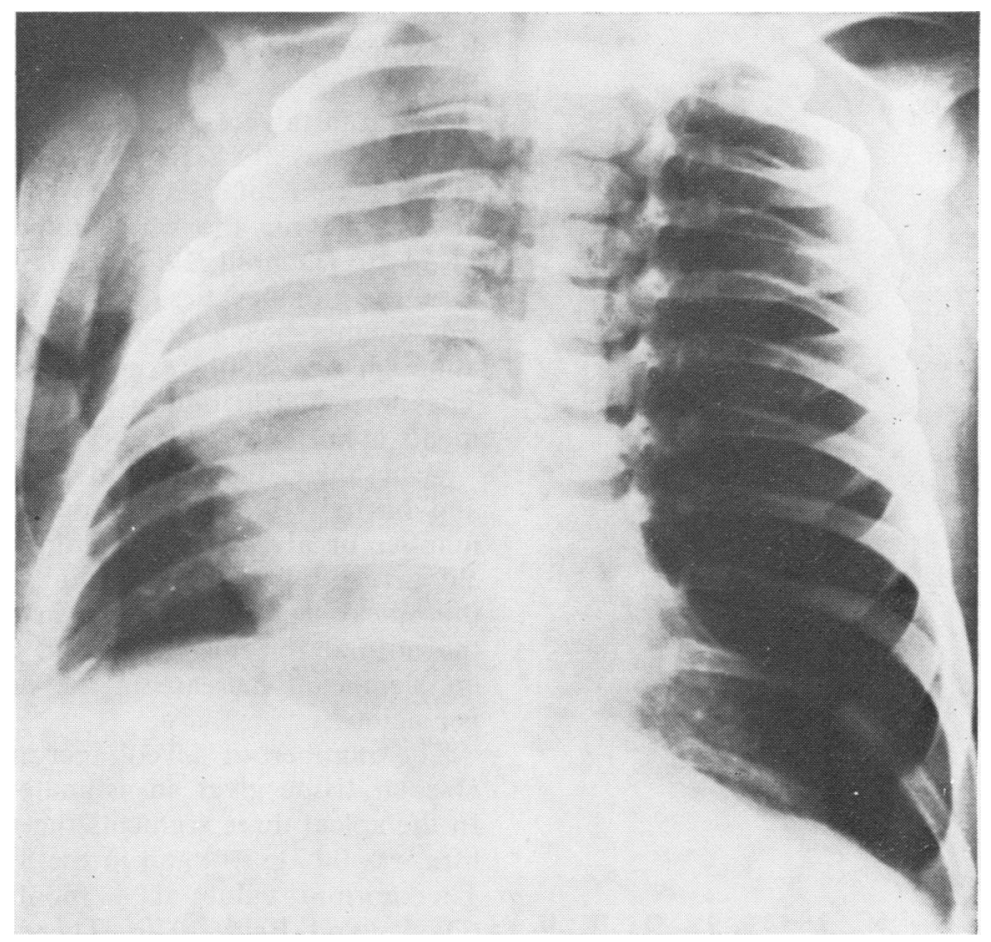

FIG. 1. Postero-anterior radiograph showing displacement of the heart and trachea to the right, with hypertransradiancy of the left lung and the left diaphragm in the normal position. The left lower lobe is more medial than is normal and the hilum is depressed, suggesting emphysema of the left upper lobe.

\section{FINDINGS}

Using the precise methods of bronchial, alveolar, and arterial quantification described in a previous paper (Hislop and Reid, 1970), it has been possible to analyse the nature of the changes in this left upper lobe.

QUANTITATIVE RESUlTS Planimetry of the posteroanterior preoperative chest radiograph (a lateral had not been taken) showed that the area of the left upper lobe was $50.7 \mathrm{~cm}^{2}$. Since the area would normally be $21 \mathrm{~cm}^{2}$, its area is increased 2.4 times and its volume $3 \cdot 7$.

MACROSCOPIC EXAMINATION AND ARTERIAL INJECTION OF SPECIMEN When the upper lobe was received its apical three segments were still inflated while the lingula appeared to have deflated normally. Figure 2 is a photograph of the freshly inflated specimen. The lingula (L) is almost completely separated from the apical three segments by a fissure and is small in comparison with the enlarged apical segments. There was no obstruction to the airways and the lobe inflated easily. The apical segments when inflated measured $14 \times 11 \mathrm{~cm}$ and were $5 \mathrm{~cm}$ thick; the lingula extended $7 \mathrm{~cm}$ from the lobar hilum.

The arteries to the anterior and apical segments were injected by the usual laboratory method with a barium gelatin mixture at $100 \mathrm{~cm} \mathrm{H}_{2} \mathrm{O}$ pressure. Injection of the upper part of the lingula was tried but was unsatisfactory because its artery had been torn. By reason of the way they had been dissected it was not possible to inject either the posterior segment or the lower half of the lingula.

The lobe was distended and fixed with $10 \%$ neutral buffered formol saline. After distension the total volume of the lobe was $270 \mathrm{ml}$. Two weeks later, when the lobe was fixed, the total volume was $168 \mathrm{ml}$. The normal total fixed volume for the left upper lobe at $2 \frac{1}{2}$ months would be about $50 \mathrm{ml}$. The lingula was dissected from the apical three segments and was found to have a volume of $28 \mathrm{ml}$, only one-sixth of that of the whole lobe, the normal proportion being two- 


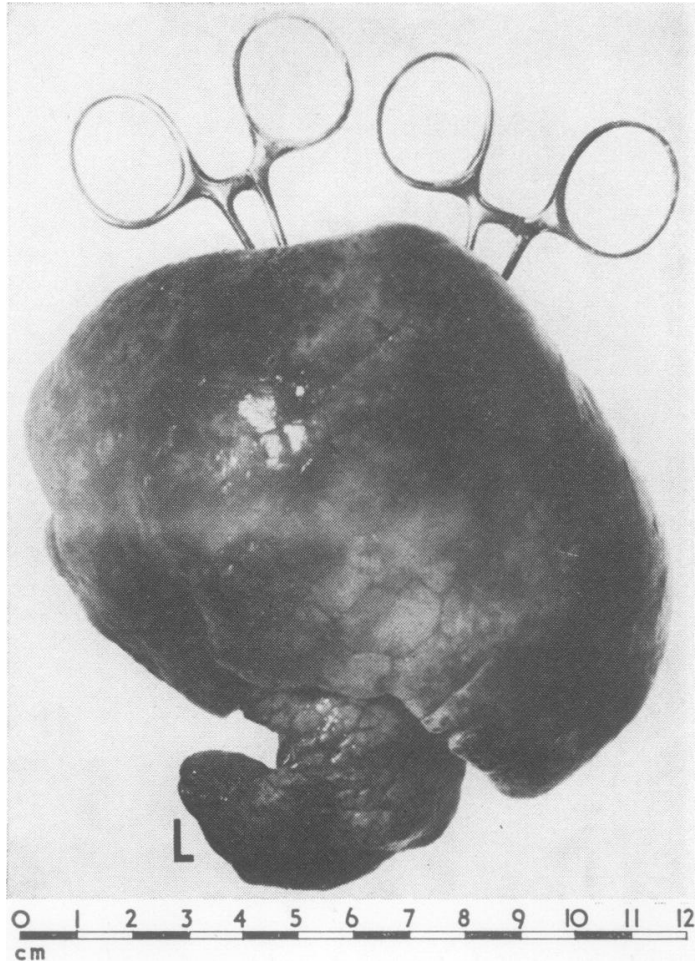

FIG. 2. Left upper lobe specimen, abnormally large after inflation with air. The lingula $(L)$ is almost separate from the apical three segments which are relatively more increased in volume.

fifths. Through the pleura the septa were clearly seen and there was some pleural vessel filling, particularly of the hilar surface. There was little evidence of emphysema and when the lobe was cut there was no evidence of retraction or increased alveolar size.

The lingula and the apical segments were sliced and point-counted separately. It was found that the 'alveolar region', i.e., structures under $1 \mathrm{~mm}$, occupied $87.25 \%$ of the apical segments and only $74 \%$ of the lingula. The normal proportion at this age is about $89 \%$ (Dunnill, 1962). Thus the alveolar region of the lingula seemed relatively reduced. Microscopic point-counting showed that the percentage of lung tissue occupied by alveoli was $52.5 \%$ in the apical segments and $48.1 \%$ in the lingula, the normal at this age being $52.5 \%$.

Alveolar region Microscopically the apical region showed enlarged alveoli but in some subpleural regions the air spaces seemed incompletely distended. The small airways also appeared dilated but with no evidence of inflammation. The lingula? had smaller alveoli than the apical region.

The total alveolar number for the apical three segments was $21.1 \times 10^{6}$ and for the lingula $7 \times 10^{6}$, giving a total of $28.1 \times 10^{6}$; the normal numberes at this age for the whole upper lobe would be $\vec{\circ}$ $21.5 \times 10^{6}$ (Dunnill, 1962). It is likely that in thiscase the number lies within the normal range. The lingula has a quarter of the total number although one would expect it to have two-fifths. $\times$ The normal distribution of alveoli between seg- $\%$ ments is not known.

Using the radial acinar alveolar count of Emery $\vec{\bullet}$ and Mithal (1960) it was found that the average number of alveoli between the respiratory bron. chiolus and the edge of the acinus was 6.2 in $\vec{z}$ the apical segments and 6.0 in the lingula. Since the normal for this age would be $5 \cdot 5$, there was no significant increase in the number of alveoli $\vec{\varphi}$ per acinus.

The number of alveoli per millilitre of fixed alveolar tissue gives an estimate of alveolar size. In the apical three segments the number per millilitre was $0.174 \times 10^{6}$ and in the lingula $0.369 \times 10^{6}$. The normal value at 4 months is $0.785 \times 10^{6} \frac{0}{8}$ (Davies and Reid, 1970). There is apparently an increase in size of alveoli in both the apical seg- $\overrightarrow{\vec{B}}$ ments and the lingula, that is, emphysema is 3 present in each though more so in the apical segments where alveolar volume is four to five times? the normal.

Bronchi Reconstruction from serial sections of $x$ the superior lingular segment showed that the $\frac{0}{3}$ number of bronchial branches was normal and therefore the number of terminal bronchioli, each with its acinus, is also normal. Cartilage was found to normal limits and the cartilage plates were normal in structure and in staining for acid glycoprotein. In the arteriogram (Fig. 3) the arteries in the anterior and apical regions appeared thin and sparse, though with the normal number and $\omega$ pattern of branches. The arteriogram of the lingula appeared abnormal because the injection medium passed within the wall as well as along the lumen. On microscopical investigation the branching pattern was found to be normal. $\frac{0}{0}$ Measurements on the arteriogram at levels along the axial pathways of the apical segment showed? that the diameter was normal for the age of theo child but was small in proportion to the lungo volume it supplied. Random sections made 


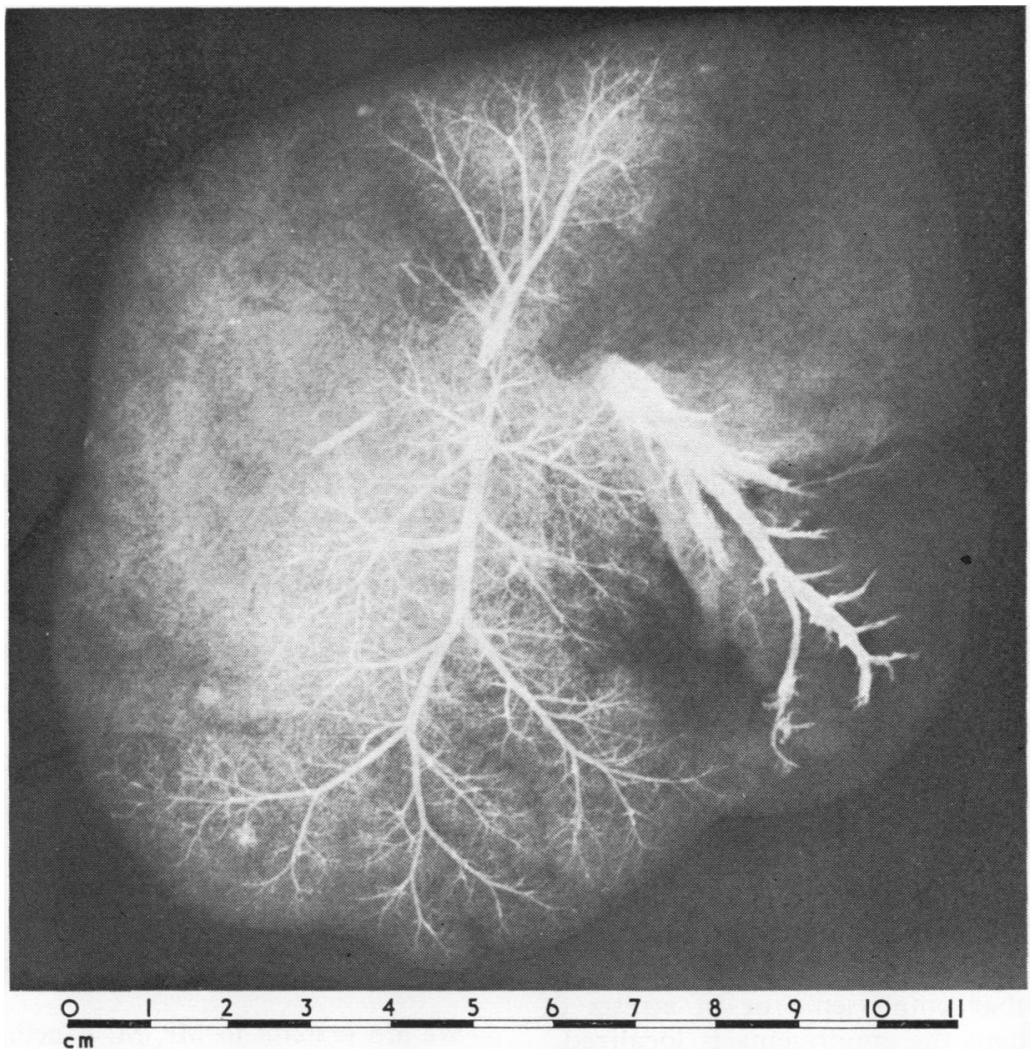

FIG. 3. Arteriogram of specimen shown in Figure 2. In the anterior and apical segments the arteries are normal in size and number. Injection of the lingula was technically unsatisfactory and artefacts were produced.

throughout the lobe showed that muscle extended to the normal level when judged by the diameter of artery and there was a normal distribution of muscular, partially muscular, and non-muscular arteries. Arteries assessed for size and structure according to their accompanying airways were normal.

There was a slight increase in the thickness of the muscle wall related to external diameter in comparison with the normal of the same age. This increase was not excessive, being about the same as that seen at birth in the normal. It was noted that there was cross-filling from the pulmonary artery circulation into the bronchial arieries. With the methods used here these anastomoses would normally be found. No precapillary pulmonary arteriovenous anastomoses were noted.

The number of arteries per unit area of alveolar region was reduced below the normal in the apical region but this might arise from the increase in alveolar size. The ratio of alveoli to arteries in the alveolar region was 6.91 in the apical region and 5.54 in the lingula, the latter representing a greater proportion of arteries, but both these can be regarded as within the normal range for this age. Since the alveoli are increased in size it seems certain that the flow per unit area of alveolar wall is reduced. Microscopically the number of capillaries per unit length of alveolar wall seemed greatly reduced.

In summary, airways and arteries in this lobe were essentially normal and the size of the arteries was appropriate for the age of the child. The alveolar count as estimated here was somewhat high but this could be explained by error inherent in the technique and by individual variation, likely to be great at an age of rapid growth.

While increase in size of artery to this lobe has occurred since birth, the persistence of the high arterial wall thickness and fetal structure 
described here may reflect a high resistance perhaps from the relative hypoxia in this lobe.

It would seem that antenatal development of the lobe was virtually normal. The significant structural change was the emphysema, that is, the increase in alveolar size throughout the lobe, but especially in the apical three segments. In view of the normal alveolar number this increase in size can be regarded as associated with a change in compliance. Any evidence of inflammation was so trivial as to be unimportant in the pathogenesis of this condition.

\section{DISCUSSION}

The abnormalities found in this lobe are the presence of emphysema and the rotation of the lobe on its hilar pedicle. Two main problems thus present for discussion - the first, whether emphysema or rotation of the lobar pedicle appeared first, and the other, how long these changes have been present.

It would seem more likely that the rotation caused the emphysema by a check-valve effect rather than the reverse, since if a localized alveolar dysplasia caused emphysema it is still necessary to explain how the emphysema would cause rotation. Rotation has not been seen in other cases of childhood lobar emphysema or in atresia of the bronchus where the emphysema is localized.

The reason for the rotation, through $180^{\circ}$ of the pedicle of the upper lobe, is by no means clear. It probably occurred after birth since the normal antenatal development of the lobe makes it unlikely that the hilum was rotated at that time. Furthermore, the surgeon was easily able to reduce the lobe to a normal position. No lymphnode enlargement at the hilum or cartilage abnormality was detected in the lobe. The possibility of a bronchial wall abnormality at, or proximal to, the level of transection cannot be excluded.

It is not easy to be certain of the stage at which the emphysema developed. It would seem unlikely that it occurred only shortly before the patient was admitted to hospital, since the child showed relatively little distress, as might have been expected if the onset was sudden. The fact that the radiographic appearance did not change over a period of several weeks also suggests that the emphysema had been present for some time. This $\bar{C}$ is further supported by the failure of the lower lobe to expand as much and as quickly as was $\frac{\bar{\rho}}{\vec{D}}$ expected immediately after resection of the upper $\stackrel{\mathbb{Q}}{\varrho}$ lobe.

The increased lobar volume did not decrease when the lobar position was corrected, suggesting that the emphysema had been present over a long $\vec{\omega}$ enough period for alveolar blood flow to have o been reduced and distensibility of the lobe to $\vec{x}$ have increased, causing irreversible emphysema. $\tilde{N}$ It is likely, therefore, that the emphysema had $\stackrel{\circ}{N}$ been present for at least some weeks before the $\vec{\theta}$ radiographic diagnosis was made. It cannot be $\oslash$ rertainly excluded that some intrinsic abnormality, 으 present even before birth, may have affected the alveoli.

The importance of this case arises from the finding of childhood lobar emphysema in a lobe $\supset$ whose antenatal development was virtually $\overrightarrow{0}$ normal. This is in striking contrast to the poly- . alveolar lobe which may present similar clinical o features. The emphysema could have followed rotation of the lobar hilum but the reason for $\bar{\partial}$ the rotation is still unexplained.

[For further discussion and bibliography, see Henderson, Hislop, and Reid, 1971.]

We are grateful to Mr. M. Paneth, Dr. M. Joseph, and Dr. G. Hinson for permission to examine this specimen and to Dr. George Simon for his opinion on the radiographs.

\section{REFERENCES}

Davies, G., and Reid, L. (1970). Growth of the alveoli and pulmonary arteries in childhood. Thorax, 25, 669.

Dunnill, M. S. (1962). Postnatal growth of the lung. Thorax, $\delta$ $17,329$.

Emery, J. L., and Mithal, A. (1960). The number of alveoli 0 in the terminal respiratory unit of man during late intrauterine life and childhood. Arch. Dis. Childh., 35, 544.

Henderson, R., Hislop, A., and Reid, L. (1971). New N pathological findings in emphysema of childhood: S 3. Unilateral congenital emphysema with hypoplasia $N$ -and compensatory emphysema of contralateral lung. N Thorax, 26, 195.

Hislop, A., and Reid, L. (1970). New pathological findings in emphysema of childhood: I. Polyalveolar lobe with $\Theta$ emphysema. Thorax, 25, 682. 